\title{
EOP's: ¿Debemos estudiar el tracto digestivo superior en pacientes con sangre oculta en materia fecal positiva y estudios del colon normales cuando realizamos rastreo de cáncer de colon?
}

\section{Caso clínico}

Durante una recorrida, un médico comenta que un paciente presentó un examen de sangre oculta en materia fecal (SOMF) positivo en el marco de un programa de rastreo de cáncer de colon. La colonoscopía fue normal y se pregunta si debe realizar estudios para evaluar el tracto digestivo superior.

\section{Pregunta que generó el caso}

En pacientes asintomáticos que presentan SOMF positiva durante el rastreo de cáncer de colon (población) los estudios para evaluar el tracto digestivo superior (intervención) detectan patología en magnitud tal que justifique su realización de rutina (resultado)?

\section{Estrategia de búsqueda}

Se realizó una búsqueda en MEDLINE empleando como términos "faecal occult blood" "upper gastrointestinal disease" "colorectal neoplasm" y "screenning"

\section{El rastreo de cáncer de colon}

El cáncer de colon es una patología frecuente, potencialmente mortal y prevenible.Su aparición antes de los 40 años es rara.La incidencia aumenta con la edad llegando a 3.7 cada mil personas por año a los 80 años. El riesgo a lo largo de la vida de padecer este cáncer es del $5 \%$. Es la tercera causa de muerte por cáncer. El cáncer de colon cumple con los criterios de Frame y Carlson ya que es una enfermedad prevalente, genera importante morbimortalidad, tiene una fase preclínica prolongada, existen métodos de diagnóstico en el período asintomático y el rastreo ha demostrado disminución de la mortalidad por este cáncer.

De allí que, con diferentes matices, todas las asociaciones científicas internacionales recomiendan incluir su rastreo en el examen periódico de salud.

Si bien la eficacia*de la intervención no genera mayor controversia, la efectividad* y la costo efectividad son temas de debate. Se sabe que la adherencia de médicos y pacientes al rastreo de cáncer de colon es inferior al $20 \%$. La costo efectividad es aceptable en países desarrollados, sin embargo la extrapolación de estos resultados a países en desarrollo no es muy clara.

A pesar de las diferentes aproximaciones con las que puede rastrearse esta patología, todas las recomendaciones dicen que si la SOMF es positiva debe evaluarse todo el colon. Lo que no aclaran las recomendaciones es cuál debe ser el seguimiento de un paciente con SOMF positiva y colonoscopía normal.

En el primer ensayo que demostró que la detección precoz con SOMF del cáncer de colon disminuía la mortalidad, el protocolo contemplaba el estudio del tracto digestivo superior. Esta práctica fue discontinuada debido al bajo rendimiento diagnóstico.

\section{Resumen de la evidencia}

Rassmussen M, Kronborg O. Upper gastrointestinal cancer in population-based screenning program with Fecal Occult Blood Test for Colorectal Cancer.Scand J Gastroenterol 2002; 37:95-98
El objetivo del estudio fue evaluar el riesgo de "pasar por alto" el diagnóstico de cáncer del tracto digestivo superior en personas con SOMF positiva en el marco de un programa de rastreo de cáncer de colon.

Este estudio incluyó a los pacientes de la rama intervención de un ensayo clínico realizado en Dinamarca para determinar si el rastreo con SOMF de cáncer de colon disminuía la mortalidad.La rama intervención tenía 30.967 pacientes entre 45 a 75 años. Hasta la fecha, estos pacientes han sido seguidos durante 15 años y han tenido ocho "rondas" de rastreo. Los pacientes asintomáticos con SOMF positiva y colonoscopía normal no recibían ningún estudio diagnóstico del aparato digestivo superior.

En 1985, 61.933 personas de entre 45 y 75 años residentes de Dinamarca, fueron invitadas a participar en un ensayo de rastreo de cáncer de colon con SOMF bienal.La determinación de SOMF se realizó con Hemoccult II. Los pacientes con SOMF positiva recibían evaluación colónica completa.

Los autores decidieron obtener información acerca de la presencia de otras neoplasias en estos pacientes de una Base de Datos y de los certificados de defunción. Se controló con el Registro Nacional de Cánceres y la Base Nacional Danesa de Pacientes. Todas las personas con diagnóstico de cáncer del tracto digestivo superior (esóf ago, estómago, intestino delgado, páncreas o vía biliar) fueron analizadas.

En el ensayo sobre rastreo de cáncer de colon, se realizaron 8 rondas de SOMF a 20.671 personas.

De las 30.967 aleatorizadas a SOMF cada dos años, 473 tuvieron una neoplasia del tracto digestivo superior en un período de 15 años (1985-2000). En 308, el cáncer fue diagnosticado entre las 20.671 que recibieron el test al menos una vez. Hubo 199 personas que tuvieron un cáncer gastrointestinal dentro de los 2 años de una SOMF negativa.

Los cánceres diagnosticados dentro de los 2 años de una SOMF positiva se describen en la siguiente tabla:

\begin{tabular}{|c|c|c|c|c|c|}
\hline Localización & $\begin{array}{l}\text { Intervalo entre } \\
\text { SOMF + y el } \\
\text { diagnóstico } \\
\text { (en años) }\end{array}$ & $\begin{array}{l}\text { Colonoscopía } \\
\text { inicial }\end{array}$ & $\begin{array}{c}\text { Síntomas en el } \\
\text { momento de } \\
\text { SOMF positiva }\end{array}$ & Anemia & $\begin{array}{l}\text { Estadío del } \\
\text { cáncer }\end{array}$ \\
\hline Esófago & 1 & Adenomas $>10 \mathrm{~mm}$ & No & No & Inoperable \\
\hline Estómago & 0 & Colon normal & Sí (FEDA) & No & Inoperable \\
\hline Estómago & 0 & Colon normal & Sí (FEDA) & Sí & Inoperable \\
\hline Estómago & 12 & Colon normal & No & No & $\begin{array}{l}\text { Operable (recaída } \\
\text { a los } 27 \text { meses) }\end{array}$ \\
\hline Estómago & 2 & Adenomas $>10 \mathrm{~mm}$ & No & No & Operable, curado \\
\hline Estómago & 14 & Adenomas $>10 \mathrm{~mm}$ & No & No & $\begin{array}{l}\text { Operable, recaída a } \\
\text { los } 6 \text { meses }\end{array}$ \\
\hline Páncreas & 8 & Colon normal & No & No & Inoperable \\
\hline Páncreas & 6 & Angiectasias & No & Sí & Inoperable \\
\hline Páncreas & 3 & Adenomas $>10 \mathrm{~mm}$ & No & No & Inoperable \\
\hline Páncreas & 6 & Cáncer Dukes A & No & No & $\begin{array}{l}\text { Operable, muerto } \\
\text { por otras causas }\end{array}$ \\
\hline $\begin{array}{l}\text { Casos Totales } \\
\mathrm{n}=10\end{array}$ & & & 2 en 10 & 2 en 10 & \\
\hline
\end{tabular}

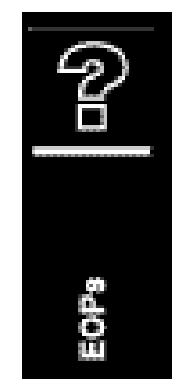

Es decir que 2 de los 10 cánceres fueron diagnosticados inmediatamente luego del test positivo por la presencia de síntomas que motivaron la realización de FEDA. Ambos fueron inoperables.

Si se tomara la presencia de anemia como indicador para investigar el tracto digestivo superior, esto habría resultado en la detección de dos cánceres. Uno tenía síntomas y fue investigado por esto por lo que hubiera sido detectado. El otro, ya era inoperable 6 meses después en el momento del diagnóstico. El resto de pacientes de la cohorte que tenían anemia (124) no tuvieron cáncer.

Los 308 cánceres detectados, 10 de los cuales se desarrollaron en personas con SOMF positiva, muestra la baja sensibilidad del 
test para neoplasias del tracto digestivo superior teniendo en cuenta que hubo 8 rondas de rastreo.

Los autores concluyen que el beneficio de realizar estudios del tracto digestivo superior en pacientes con SOMF positiva y colon normal en el marco de rastreo para cáncer de colon, sería muy pequeño y muchos los estudios necesarios.

Por lo tanto, estudios del tracto digestivo superior no deberían realizarse en programas de rastreo con Hemoccult II para detección de cáncer de colon en personas con riesgo habitual cuando la SOMF es positiva y la colonoscopía normal a menos que la persona esté sintomática.

\section{Comentario}

La eficacia* del rastreo de cáncer de colon está claramente establecida. Su efectividad* y costo efectividad especialmente en países en desarrollo es materia de debate.

De implementarse, nos encontraríamos con varias situaciones que nos generarían dudas: ¿cómo seguir a los pacientes con pólipos colónicos?, ¿qué hacer si la SOMF es positiva y la colonoscopía normal?.

El estudio resumido, pretende responder la segunda pregunta. Entonces, ¿podemos quedarnos lo suficientemente tranquilos en una persona con SOMF positiva cuya evaluación colónica es normal?

En principio, la respuesta parece ser sí, pero debemos tener en cuenta algunas consideraciones.
1) El test empelado en este estudio fue el Hemoccult II (guayaco) por lo que estas conclusiones no necesariamente se aplican a otros métodos diagnósticos

2) La población estudiada no tiene alta prevalencia de cáncer de tracto digestivo superior y las conclusiones no pueden extrapolarse a pacientes de alto riesgo (ejemplo japoneses)

3) La selección de la muestra fue hecha para un programa de rastreo de cáncer de colon (es población general en su enorme mayoría asintomática) y esto debe tenerse en cuenta ya que la conducta ante SOMF positiva podría variar si el paciente realiza el test por algún otro motivo.

El resultado de este estudio concuerda con otros análisis retrospectivos 1 de pacientes con SOMF positiva y con lo hallado en el estudio de Minnessota2 en el cual entre 1975 y 1982 realizaron FEDA a los pacientes con SOMF positiva. Luego de ese período, se decidió discontinuar esa práctica debido a la bajísima prevalencia de patología pasible de tratamiento.

\section{Conclusión}

Los estudios del tracto digestivo superior no deberían realizarse en programas de rastreo para detección de cáncer de colon en personas con riesgo habitual cuando la SOMF es positiva y la colonoscopía normal a menos que la persona esté sintomática.

Dra. Karin Kopitowski [ Unidad de Medicina Familiar y Preventiva. Hospital Italiano de Buenos Aires ]

\section{Referencias}

1 Chen YK, Gladden DR, Kestenbaum DJ et al.Is there a role for upper gastrointestinal endoscopy in the evaluation of patients with occult blood-positive stool and negative colonoscopy? Am J gastroenterol 1993; 88:2026-9.

2 Mandel JS, Bond JH, Church TR, et al.Reducing mortality from colorectal cancer by screening for fecal occult blood. Minnesota Colon Cancer Control Study.N Eng J Med 1993; 328:1365-71.

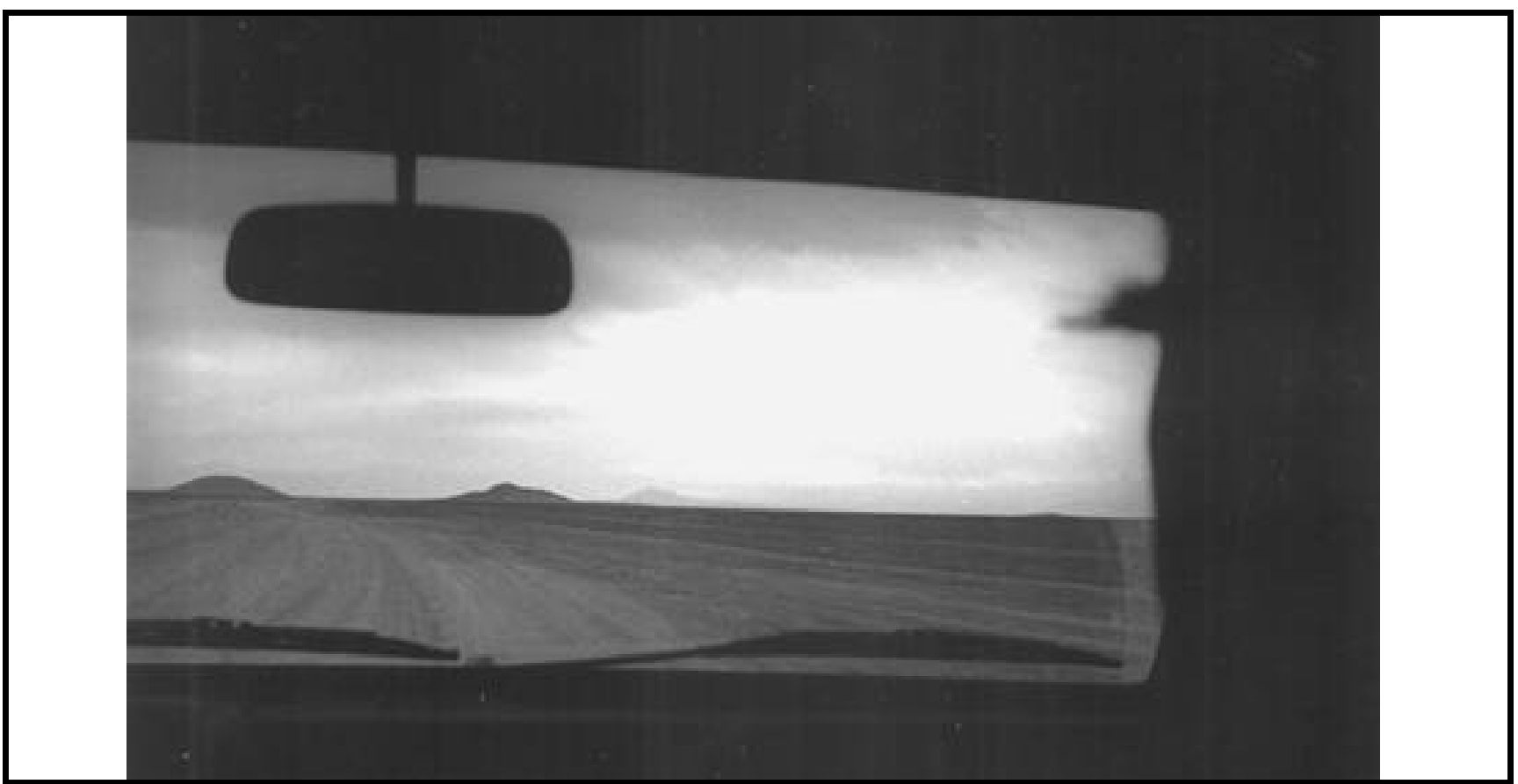

